\title{
Musculoskeletal ultrasound versus MRI of the hands in healthy subjects - a pilot study
}

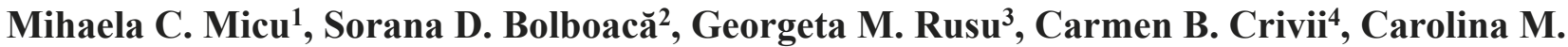 \\ Solomon 5
}

${ }^{1}$ Rheumatology Division, $2^{\text {nd }}$ Rehabilitation Department, Rehabilitation Clinical Hospital, ${ }^{2}$ Department of Medical Informatics and Biostatistics, "Iuliu Haţieganu" University of Medicine and Pharmacy, ${ }^{3}$ Radiology Department, Emergency Clinical County Hopsital, ${ }^{4}$ Morphology Department, "Iuliu Haţieganu" University of Medicine and Pharmacy, ${ }^{5}$ Department of Radiology, „Iuliu Haţieganu” University of Medicine and Pharmacy Cluj-Napoca, Romania

\begin{abstract}
Aim: High resolution imaging methods detect a spectrum of inflammatory-like and structural modifications at joint and tendon level in healthy subjects. The knowledge of their extent and degree is important when subclinical disease activity (implying therapy reassessment) must be differentiated from normality. Musculoskeletal ultrasound (MSUS) evaluation may be challenging even for experts when borderline or low grade lesions are present. Our objective was to analyse the frequency of inflammatory-like lesions in hand joint and tendons in healthy young subjects and to evaluate the concordance between MSUS and magnetic resonance imaging (MRI) findings. Material and methods: Ten healthy young women (age range 24-32 years) clinically asymptomatic (joints and tendons) were selected to have bilateral hand MSUS and MRI evaluation. Based on current definitions, synovitis/tenosynovitis-like lesions, erosions, osteophytes and bone edema were quantified and concordance between the two imaging methods was calculated. Results: Overall, both imaging evaluation methods showed a low frequency of inflammatory-like and structural lesions. No joint presented power Doppler signal or erosions. No abnormalities suggestive for inflammatory or structural pathology were detected at the tendon compartments level. No erosions and no signs of osteitis were detected. The concordance between MSUS and MRI findings was high except for the wrist area. Conclusion: MSUS was demonstrated to be a very accurate imaging method, mostly for hand tendon evaluation. This would allow a better discrimination between normality and pathologic findings, adding supplementary information.
\end{abstract}

Keywords: musculoskeletal ultrasound; Magnetic Resonance Imaging (MRI); joints; tendons; healthy subjects

\section{Introduction}

The border between imaging spectrum of normality in joints and tendons and subclinical pathology in rheumatoid arthritis (RA) is still under debate. Treatment readjustment represents a big challenge for practitioners when subclinical mild inflammatory lesions are detected.

Received 28.10.2018 Accepted 17.01.2019

Med Ultrason

2019, Vol. 21, No 2, 117-124

Corresponding author: Carmen B. Crivii

Morphology Department, "Iuliu Haţieganu"

University of Medicine and Pharmacy

Cluj-Napoca, 3-5 Clinicilor street,

400006, Cluj-Napoca, Romania

E-mail: bianca.crivii@umfcluj.ro
Clinical and imaging remission reflects a different dimension of normality, the dissociation between the two being highlighted in several studies [1-3]. Imaging studies on RA patients in clinical remission, overlapping or not with patients' opinion for RA remission, certify the presence of residual inflammatory findings in joints as well as in tendons [1-21]. Among high resolution imaging methods, musculoskeletal ultrasound (MSUS) was demonstrated to be more suitable for clinical practice and studies, allowing more data collection in terms of patient numbers, in comparison to magnetic resonance imaging (MRI). Indeed, it has been shown to be a very attractive imaging tool because of its accuracy and feasibility in early as well as in longstanding disease [22-24].

Several MSUS studies focusing on healthy subjects identified the presence of grey scale inflammatory pathol- 
ogy-like findings (mild, prevalent effusion), mostly in the wrists (dominant hand) and rare in metacarpophalangeal joints (MCPj) or proximal interphalangeal joints (PIPj). These modifications were correlated with biomechanical factors and age [25-31]. Instead, tenosynovitis/tenosynovitis-like modifications and Power Doppler signal (PDUS) at joint and tendon level were depicted very rarely in healthy populations [32-35]. Recently, a longitudinal study on healthy women focused attention especially on flexor tendons in the hands, $\mathrm{MCPj}$ and extensor carpi ulnaris tendon (ECU) that seem to have a very low frequency of modifications in the younger healthy population. These structures were found to show stability in time when exposed to different factors linked to pregnancy status/ postpartum period [36].

Hands MRI studies in healthy subjects identified a low prevalence of true low-grade synovitis or synovitislike changes (minimal early synovial enhancement) in $\mathrm{MCPj}$ and wrists. Osteophytes, small bone erosions and bone marrow edema were occasionally found. In contrast, a common finding was tendon sheath effusion identified on MRI without contrast [29,31,32,34,37].

Only one study evaluating MCPj in RA patients included a low number of controls which were evaluated by both imaging methods - MSUS versus contrast MRI, showing no synovial membrane thickening on greyscale MSUS, no intra-articular power Doppler (PD) signal and no or only slight synovial enhancement on dynamic MR images [27]. By now, no comparative MSUS vs MRI study in healthy subjects focusing on hand tendons was performed.

Our objective was to analyse the frequency of inflammatory-like lesions in hand joint and tendons in healthy young subjects and to evaluate the concordance between MSUS and MRI findings. The main focus was addressed to the wrist area, MCPj 2-5, ECU and flexor tendons.

\section{Material and methods}

This was a prospective cross-sectional study performed in May 2018. Ten healthy young women, age $>18$ years, clinically asymptomatic, with low up to moderate level of physical effort were selected. Exclusion criteria were: age $>35$ years, diagnosis of a current acute/subacute or chronic inflammatory/autoimmune rheumatic disease, history of NSAIDs/painkiller administration in the last 6 weeks, history of trauma in the hands in the last 2 years, intense physical activity involving hands (hard work/sport). All participants signed the informed written consent prior enrolment and local Ethical Committee approval was obtained.

Following demographic data were collected: type of profession, type of physical work/sport involving hands, dominant hand, personal and family medical history, current medication other than specified in the exclusion criteria, smoking, body mass index (BMI). Clinical, MSUS and MRI evaluation of the hands was done in an interval of maximum 72 hours, by a senior doctor with $>10$ years of experience.

\section{Clinical examination}

Clinical examination (CE) was focused on the following anatomic regions and structures: wrist, $\mathrm{MCPj} 1-5$, PIPj 1-5, bilateral hand extensor tendon compartments $1-6$, flexor pollicis longus tendon (FPL) and superficial and profound flexors of the digits 2-5 (F2-5) of both hands. The absence of any pain in active/passive motion along with a normal range of motion qualified the subject for the study.

\section{MSUS examination}

MSUS evaluation of both hands was performed on the same day as CE, according to current guidelines [22], in the same regions as CE. If present, following elementary lesions were recorded: joint synovial hypertrophy/effusion (SH/E) by grey scale (GS), intra-articular PD signal, erosions, osteophytes, $\mathrm{SH} / \mathrm{E}$ and intra-tendon sheath and/ or intra-tendon belly PD signal. The OMERACT definitions for synovitis, tenosynovitis, effusion, erosion and osteophytes were used [38,39]. Joint and tendon pathology was quantified with a semi-quantitative scoring system for small joints [40] and tendons [39]. For erosions and osteophytes, a dichotomous (normal or abnormal) scoring system was used. Dorsal and volar scoring of each MCPj and PIPj were merged into a single scoring/ joint. If dorsal and volar scoring of one joint were different (one was normal and other was abnormal) the abnormal finding for $\mathrm{SH}, \mathrm{E}$ and $\mathrm{PD}$ was chosen to be recorded as a single elementary lesion. Tendon examination was performed in a multi-plane (at the level of the extensor retinaculum for $\mathrm{C} 1-\mathrm{C} 6$ to the distal insertion and from the level of the flexor retinaculum up to the distal insertion for FPL, F2-F5) and dynamic fashion, according to specific manoeuvres. Lateral sites of MCPj 2 and 5 were also evaluated for erosions.

A Samsung RS 85 machine equipped with a 3-16 $\mathrm{MHz}$ broadband multi-frequency linear transducer was used. GS settings were adjusted in a standardized manner for superficial anatomic regions. The settings for PD examination were low filter wall and pulse repetition frequency for small vessels with slow flow $500-750 \mathrm{~Hz}$.

\section{MRI evaluation}

A General Electric Sigma Explorer scanner 16 channels, 1.5-T, with dedicated hand protocol was used.

The following MRI 2D, without gadolinium administration, sequences were acquired: STIR (Short Tau Inversion Recovery), T2 MERGE (Multiple Echo Re- 
combined Gradient Echo) and T1 FSE (Fast Spin Echo). STIR was performed in axial plane; slices perpendicular on the metacarpal and phalangeal bones, included the radio-ulnar joint and the distal phalangeal bone of $3^{\text {rd }}$ finger. T2 MERGE and T1 FSE in coronal plane covered the whole region, from dorsal to the palmar aspect. Slices were obtained parallel to the metacarpal and phalangeal bones. The same joint/tendon set as in MSUS evaluation was assessed together with following bones: distal radius epiphysis, carpal bones, digit 1-5 metacarpal, proximal and distal phalanx bones. Acquisition parameters are shown in Table I.

All MR images were assessed by a trained musculoskeletal radiologist with more than 14 years of experience who was blinded to clinical details. The following parameters were assessed based on the Outcome Measures in Rheumatology Clinical Trials (OMERACT) Rheumatoid Arthritis MRI Scoring System (RAMRIS): joint synovitis, bone marrow edema, bone erosions [41]. According to the present MRI protocol, without contrast, only joint capsule distension was possible to be identified. Absence of fluid was evaluated with 0 and fluid-like capsule distension (mild, moderate and severe) with 1,2, and 3 .

The fluid-like signal inside the tendon sheaths was measured according to a previous MRI protocol described [42] at the point of maximal thickness, perpendicular to the tendon surface. Bone marrow edema and erosions were noted when present or absent. Intra-osseous cysts were defined as sharply demarcated hyper-intense lesions within the bone on any fluid-sensitive MRI sequences, visible on two planes, without destruction of the overlaying cortical bone. Cysts were quantified using a dichotomous score.

\section{Statistical analysis}

Data were summarized according to their type. The quantitative data were presented as a median and interquartile range $(\mathrm{IQR}=\mathrm{Q} 1-\mathrm{Q} 3$, where $\mathrm{Q} 1=$ first quartile, $\mathrm{Q} 3$ = third quartile). Absolute frequencies expressed as the number of cases with a specific characteristic divided to the number of possibilities were used to summarize the presence of effusion (E), synovial hypertrophy ( $\mathrm{SH}$ ), erosion (ER), and respectively osteophyte (O). The concordance between MSUS and MRI findings was evaluated by dividing the number of agreements to the number of cases and the percentages were reported associated with the $95 \%$ confidence interval [43].

\section{Results}

Ten healthy volunteers, all women, age between 24 and 32 years (median 29, IQR [26.00-31.25]) were included. All participants were right handed, half of them showing history of moderate physical effort. One participant had Hashimoto thyroiditis. Family history of RA was present in two participants and skin psoriasis and morfea in two other participants.

Most participants had normal body mass index (8/10), while two of them were obese (BMI range from 18 $\mathrm{kg} / \mathrm{m}^{2}$ to $28 \mathrm{~kg} / \mathrm{m}^{2}$, median of $19 \mathrm{~kg} / \mathrm{m}^{2}$ and IQR (19 to $22)$. Four out of 10 participants were smokers ( $\leq 8$ cigarettes/day).

Overall, a low frequency of inflammatory-like and structural lesions were identified. MSUS evaluation identified $\mathrm{SH}$ in $7 / 20(35 \%, 95 \% \mathrm{CI}[15.25$ to 59.75$])$ wrist joints, with distribution in both hands (4 of grade 1 and 3 of grade 2), grade 1 effusion in $6 / 80(7.5 \%, 95 \% \mathrm{CI}$ [2.52 to 14.98]) MCPj 2-5, one joint having in addition SH grade $1(1.25 \%)$. Osteophytes were detected in $2 / 80$ (2.5\%, 95\%CI [0.02 to 8.73]) $\mathrm{MCPj} 2-5$ and in 40/100 $(40 \%, 95 \% \mathrm{CI}$ [30.01 to 49.99]) of the PIPj 1-5. No joint presented PD signal or erosions. In one subject, a nutritive vessel at the level of the $3^{\text {rd }}$ metacarpal head was detected. No abnormalities were detected at the tendon compartments level. Dynamic tendon evaluation was asymptomatic and showed a smooth gliding of the tendons along with a normal joints range of motion. A small amount of fluid/fluid-like material, grade $1(<1.5 \mathrm{~mm}$, range 0.3-1.2 mm, mostly concentric) was detected inside the flexor tendon sheaths in several locations, in all participants, with the highest amount detection at MCPj level.

MRI evaluation identified very small quantities of effusion (grade 1) at the level of the MCPj 1 in $2 / 20(10 \%$, $95 \% \mathrm{CI}[1.00$ to 59.00$]), \mathrm{MCPj} 2-5$ in $5 / 80(6.25 \%, 95 \% \mathrm{CI}$ [2.52 to 13.73$])$, osteophytes in the $1 / 80(1.25 \%, 95 \% \mathrm{CI}$ [0.02 to 6.23]) MCPj 2-5 and 16/100 (16\%, 95\%CI [9.01 to 24.99]) in the PIPj 2-5. No inflammatory-like pathol-

Table I. MRI acquisition parameters used

\begin{tabular}{|c|c|c|c|c|c|c|c|c|c|}
\hline $\begin{array}{l}\text { Plane and acquisition } \\
\text { sequence }\end{array}$ & $\begin{array}{l}\text { TR } \\
(\mathrm{ms})\end{array}$ & $\begin{array}{l}\text { TE } \\
(\mathrm{ms})\end{array}$ & $\begin{array}{l}\text { TI } \\
(\mathbf{m s})\end{array}$ & $\begin{array}{l}\text { FA } \\
\text { (degrees) }\end{array}$ & $\begin{array}{l}\text { FOV } \\
\text { (cm) }\end{array}$ & $\begin{array}{l}\text { Slice thickness } \\
(\mathrm{mm})\end{array}$ & $\begin{array}{l}\text { Interslice } \\
\text { gap (mm) }\end{array}$ & $\begin{array}{l}\text { Matrix (freq/ } \\
\text { phase) }\end{array}$ & $\begin{array}{l}\text { Pixel size } \\
(\mathrm{mm})\end{array}$ \\
\hline Axial STIR & $5400-5500$ & $42-44$ & 120 & 160 & 15 & 3 & 0.3 & $288 / 192$ & $0.5 \times 0.8$ \\
\hline Coronal T2 MERGE & $450-520$ & 15 & & 20 & 21 & 2 & 0.2 & $256 / 224$ & $0.8 \times 0.9$ \\
\hline Coronal T1 FSE & $500-600$ & $13-15$ & & 160 & 21 & 2 & 0.2 & $412 / 224$ & $0.5 \times 0.9$ \\
\hline
\end{tabular}

TR - Repetition Time, TE - Echo Time, TI - Inversion Time, FA - Flip Angle, FOV - Field of View 
Table II. Summary and distribution of grey scale ultrasound (US) findings vs. magnetic resonance imaging (MRI) findings

\begin{tabular}{|c|c|c|c|c|c|c|c|c|}
\hline & \multicolumn{4}{|c|}{ US no/n } & \multicolumn{4}{|c|}{ MRI no/n } \\
\hline & $\mathrm{E}$ & $\mathrm{SH}$ & ER & $\mathrm{O}$ & $\mathrm{E}$ & $\mathrm{SH}$ & ER & $\mathrm{O}$ \\
\hline \multicolumn{9}{|l|}{ Joints } \\
\hline Wrist & $0 / 20$ & $7 / 20$ & & $0 / 20$ & $0 / 20$ & $0 / 20$ & & $0 / 20$ \\
\hline MCP 1 & $0 / 20$ & $0 / 20$ & & $0 / 20$ & $2 / 20$ & $0 / 20$ & & $1 / 20$ \\
\hline MCP 2-5 & $6 / 80$ & $1 / 80$ & $0 / 40$ & $2 / 80$ & $5 / 80$ & $0 / 80$ & $0 / 40$ & $1 / 80$ \\
\hline PIP 1-5 & $0 / 100$ & $0 / 100$ & & $40 / 100$ & $3 / 100$ & $0 / 100$ & & $16 / 100$ \\
\hline \multicolumn{9}{|l|}{ Tendons } \\
\hline $\mathrm{C} 1-\mathrm{C} 5$ & $0 / 100$ & $0 / 100$ & & & $0 / 100$ & $0 / 100$ & & \\
\hline $\mathrm{F} 1-\mathrm{F} 5$ & $0 / 100$ & $0 / 100$ & & & $0 / 100$ & $0 / 100$ & & \\
\hline ECU & $0 / 20$ & $0 / 20$ & & & $0 / 20$ & $0 / 20$ & & \\
\hline
\end{tabular}

MCP - metacarpophalangeal, PIP - proximal interphalangeal, C1-C5 - extensor tendon compartments 1-5, F1-F5 - flexor tendons of the digits 1-5, ECU - extensor carpi ulnaris tendon, E - effusion, SH - synovial hypertrophy, ER - erosion, O - osteophyte, no - number of findings, $\mathrm{n}$ - number of areas.

ogy was detected at wrist level. No erosions and no signs of osteitis were detected. Several subchondral cysts were detected at carpal bones and metacarpal heads level (11 - lunate, 3 - trapezium, 3 - trapezoid, 8 - capitate bone, 1 - hamate, 3 - triquetrum, 4 - pisiform, 1 - MC1, 4 - MC2, 4 - MC3, 1 - MC4). Effusion inside the tendon sheath (grade 0 , mean of $0.8 \pm 0.34 \mathrm{~mm}$ ) was detected in $8(8 \%$, $95 \%$ CI [3.01 to 14.99]) tendon units- flexor tendons (4 $\mathrm{F} 2,1 \mathrm{~F} 3,3 \mathrm{~F} 4)$ and one $(1 \%) \mathrm{C} 2$, in 5 out of 10 subjects, mostly unilateral. Table II presents the summary and distribution of the grey scale lesions MSUS vs MRI.

The overall concordance between US findings and MRI findings was evaluated whenever the results presented in Table II were discordant. Results are presented in Table III.

The lowest concordance between MSUS and MRI was found in wrist (65\%) and PIP for osteophytes (72\%). MRI detected a number of $5 \mathrm{MCPj} 2-5$ with effusion ver-

Table III. Details of concordance between US findings and MRI findings

\begin{tabular}{lll}
\hline Area & \multicolumn{2}{l}{ Concordance } \\
\cline { 2 - 3 } & $\mathbf{n o / n}$ & $\mathbf{\%}[\mathbf{9 5 \%} \mathbf{n} \mathbf{C I}]$ \\
\hline Wrist SH & $13 / 20$ & $65.00[40.25$ to 84.75$]$ \\
MCP 1 E & $18 / 20$ & $90.00[70.25$ to 99.75$]$ \\
MCP 1 O & $19 / 20$ & $95.00[75.25$ to 99.75$]$ \\
MCP 2-5 SH & $79 / 80$ & $98.75[91.27$ to 99.98$]$ \\
MCP 2-5 E & $69 / 80^{*}$ & $86.25[76.27$ to 92.48$]$ \\
MCP 2-5 O & $77 / 80^{*}$ & $96.25[90.02$ to 98.73$]$ \\
PIP O & $72 / 100^{* *}$ & $72.00[62.01$ to 80.99$]$ \\
PIP E & $97 / 100$ & $97.00[92.01$ to 98.99$]$ \\
\hline
\end{tabular}

SH - synovial hypertrophy, E - effusion, O - osteophyte, MCP - metacarpophalangeal, PIP - proximal interphalangeal, no - the number of concordances, $\mathrm{n}$ - total number of the evaluated structure, $*$ none of the effusion identified by MSUS was identified by MRI and none of the effusion identified by MRI was identified by MSUS, ** in 14 cases the osteophytes were identified by both MSUS and MRI sus 6 effusions detected on MSUS, showing high concordance among the methods. Flexor tendon evaluation and ECU showed $100 \%$ concordance MSUS vs MRI.

In figure 1 there are some findings encountered during MSUS and MRI examinations.

\section{Discussions}

Our study is the first to compare MSUS with MR findings in healthy subjects at the hand level. The concordance calculation between the two methods raised several issues to be clarified at the wrist, MCPj and tendon level.

Concordance for tendon findings was $100 \%$, confirming that multi-plane and dynamic USMS evaluation identifies with high accuracy the normal aspect of these structures. In addition, we confirmed with both methods that young healthy subjects, performing moderate daily hand effort, present no grey scale or Doppler pathology at tendon level. Our focus was especially oriented on flexor tendon since a previous MSUS study performed in pregnant versus non-pregnant healthy women ( $n=20$ vs $n=75$ ) confirmed the absence of hand flexor tendon pathology in both groups, cross-sectional and longitudinal [36].

Tenosynovitis is a complementary, but different facet of the inflammatory involvement in RA and is present from early disease stages [44-46]. In this sense, imaging evaluation could add important information to the algorithm increasing the specificity of the new ACR/EULAR classification criteria for RA, especially for early phases $[44,47,48]$.

In order to understand how to include tendon inflammatory pathology in our clinical algorithm it is important to know about the frequency of such abnormalities in healthy subjects.

Our findings are in line with other studies focusing on tendon evaluation in healthy subjects or including 


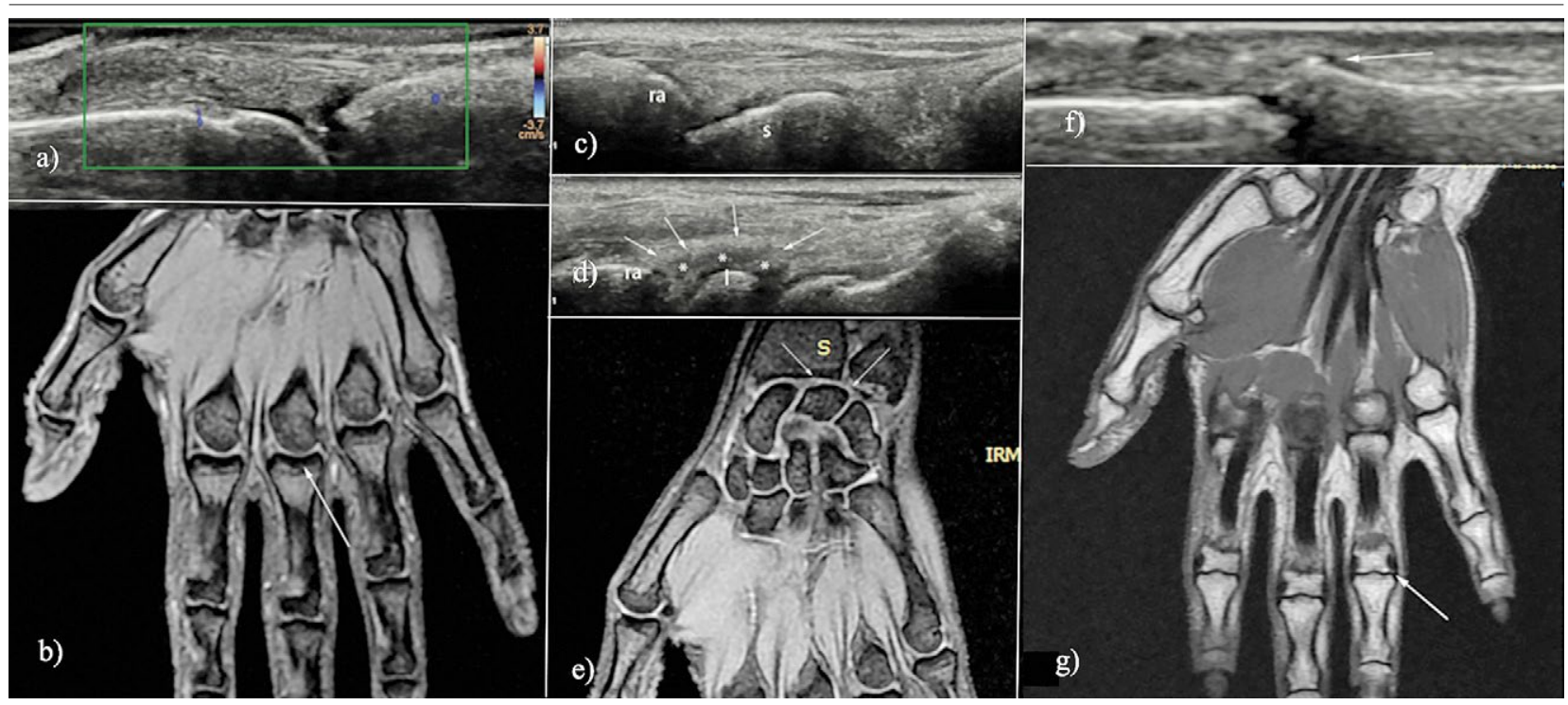

Fig 1. Female, 32 years old, 3rd MCP joint: a) longitudinal ultrasound with detection of a nutrient vessel; b) MRI, coronal plane, 2D MERGE sequence- no effusion inside the joint. Female, 26 years old, radiocarpal joint: c) longitudinal ultrasound, normal aspect between proximal radius (ra) and distal scaphoid bone (s); d) hypoechoic material distending the capsule (arrows) between proximal radius (ra) and distal lunate bone (l); e) MRI, coronal plane, 2D MERGE sequence, right hand - no pathological fluid at the radiocarpal joint, especially at lunate bone level (arrows). Female 32 years old, PIP joint: $f$ ) longitudinal ultrasound showing the sharp bony cortex- small osteophyte (arrow); g) MRI, coronal plane, T1 sequence - small osteophyte (arrow).

healthy control groups, showing a very low prevalence of pathologic findings, mostly unilateral, which correlates probably more with age and intense physical activity involving hands. A prospective MRI study focusing on the hand structures evaluation in 23 healthy subjects (range of 25-86 years) found out that only $4.3 \%$ (1 subject) presented extensor tendon involvement vs $17.4 \%$ (4 subjects) showing flexor tenosynovitis. In this study, the subjects were much older compared with our subjects [29].

Another study compared the prevalence of tenosynovitis in the hands in RA patients vs 20 controls. Tenosynovitis of the flexor tendons was present in $65 \%$ of patients vs only 1 of the $20(5 \%)$ controls/1 tenosynovitis [32]. No tenosynovitis of the flexor tendons was found in the control group $(\mathrm{n}=10)$ of another study that addressed tenosynovitis as an important MRI finding in early RA [48]. In both these studies, the participants were older than in our study and tenosynovitis in the control group was assessed on gadolinium-enhanced MRI images.

Wakefield et al analysed a group of not treated early RA patients using MSUS and MRI. They found a frequency of $28 \%$ vs $64 \%$ digit flexors tenosynovitis and $14 \%$ vs $40 \%$ for the extensors, in favor of MRI evaluation. No tenosynovitis was detected in the control group of health subjects but they underwent only MSUS evaluation [49].

The largest prospective MRI study performed on 42 healthy volunteers identified fluid inside the tendon sheath as being a very common finding in at least 1 location. The authors concluded that fluid in the finger flexor tendon sheaths may be a normal finding and that one should be cautious when interpreting these findings as pathology in MRI images without gadolinium administration [31].

In our study we performed in each tendon a multiplanar and dynamic MSUS evaluation. All tendons that were evaluated as normal, not only fulfilled the morphologic normality criteria but showed also a smooth gliding pattern of the tendon in relation to the surrounding structures, with a normal range of motion; PD signal was absent in all evaluated tendons. Generally, MSUS information obtained during dynamic manoeuvre scan may be of great value when differentiating true inflammatory pathology from imaging inflammatory-like pathology. A truly inflamed tendon will show gliding abnormalities together with a variable spectrum of morphologic modifications in the tendon sheath/tendon belly. MSUS evaluation has this advantage over MRI where only morphologic information can be depicted [39,50]. At this point we could hypothesize that tenosynovitis could be a very feasible discriminative imaging parameter between healthy subjects and groups with subclinical disease.

At wrist joint level we faced the lowest concordance between the two imaging methods. MSUS depicted SH (grade 1 or 2, without PD signal) in 7/20 (35\%) wrists, none being confirmed by MRI evaluation. This 
result raised the problem of wrist $\mathrm{SH}$ overestimation with MSUS, mostly in situations when the transducer is placed between the radius and lunate bone. The presence inside the joint space of hypoechoic inhomogeneous material distending the capsule may be interpreted as $\mathrm{SH}$. A possible confounder could be represented by synovial folds being pushed inside the joint recess. So, in absence of any capsular distension visible at radio-scaphoid level, in absence of associated effusion or PD signal at this level one should be cautious in interpreting these modifications as MSUS radiocarpal synovitis.

MCPj evaluation was provocative. Concordance between MSUS and MRI in detecting joint effusion (grade 1 in both scoring systems) was high but joint by joint analysis revealed a total discordance between the findings' location. We concluded at this point that effusion grade 1 may be a challenge even for an experienced MSUS performer. More objective MRI findings could be explained by detecting these small quantities of effusion in the extreme lateral areas of the joint recess, a place with less accessibility for MSUS evaluation.

Regardless of the imaging method used, several other studies identified a low frequency of mild MCPj pathology (SH and/or E), mostly unilateral, sometimes associated with a low grade PDUS [25-30,34-37,40].

Detection of intra-articular nutrient vessels may generate a false interpretation for the PD signal inside the joint. It is well known by now that subclinical PD positive synovitis is linked to further joint destruction in clinical remission RA patients [2]. In fact, PD signal is encountered more often in joints that show erosions [51]. Therefore, in joints without grey scale $\mathrm{SH}$, presence of true PD signal must be carefully analysed [52].

We detected no erosion and no bone marrow edema in any location suggesting that in very young healthy people these findings are absent in comparison to older healthy groups $[26,27,34,52]$. Osteophytes showed a lower concordance between the two imaging methods. MSUS detected very small osteophytes in $40 \%$ PIPj vs $16 \%$ detected by MRI. Firstly, we could conclude that isolated osteophytes start to be present in very young healthy population (the same trend was observed also in cortical cysts). Secondly, the very sharp phalangeal bony cortex could be misinterpreted at MS US as an incipient osteophyte.

Our study has the advantage of comparing for the first time two high resolution imaging methods in healthy subjects offering a more objective mirror regarding possible imaging abnormalities. Indeed, the discussions were focussed on potential pitfalls to be encountered/avoided in clinical practice as well as in trials.
The limits of the study were the low number of participants, suitable only for a pilot study and the MRI protocol which did not include contrast agents. Contrast enhanced MRI evaluation has shown to be superior to the native one by detecting with a high accuracy synovial hypertrophy and differentiating it from effusion, both in joints and tendons. Because of ethical reasons we did not enrol healthy subjects for this kind of protocol, several studies showing a risk for potential side effects [53]. Another limit would be the absence of an interobserver reliability exercise, both for MSUS and MRI.

In conclusion, MSUS has been demonstrated to be a very accurate imaging method mostly for hand tendon evaluation. This will allow a better discrimination between normal and pathologic findings, adding supplementary information. In the young population, flexor tenosynovitis coupled with wrist/MCPj synovitis should trigger the practitioners' attention for early disease detection or future flare recognition.

Acknowledgements: We would like to thank to Oana Șerban, Bianca Bălan, Iulia Papp, Bianca Pop, Andreean Relenschi, Linda Inocan, Alexandra Cherecheș, Iulia Moldovan, Jessica Trif and Mihaela Coștișor for their valuable contribution in performing this study.

\section{References}

1. Brown AK, Quinn MA, Karim Z, et al. Presence of significant synovitis in rheumatoid arthritis patients with diseasemodifying antirheumatic drug-induced clinical remission: evidence from an imaging study may explain structural progression. Arthritis Rheum 2006;54:3761-3773.

2. Brown AK, Conaghan PG, Karim Z, et al. An explanation for the apparent dissociation between clinical remission and continued structural deterioration in rheu- matoid arthritis. Arthritis Rheum 2008;58:2958-2967.

3. Picchianti Diamanti A, Navarini L, Messina F, et al. Ultrasound detection of subclinical synovitis in rheumatoid arthritis patients in clinical remission: a new reduced-joint assessment in 3 target joints. Clin Exp Rheum 2018;36:984989.

4. Kawashiri SY, Suzuki T, Nakashima Y, et al. Ultrasonographic examination of rheumatoid arthritis patients who are free of physical synovitis: power Doppler subclinical synovitis is associated with bone erosion. Rheumatology (Oxford) 2014;53:562-569.

5. Molenaar ET, Voskuyl AE, Dinant HJ, Bezemer PD, Boers M, Dijkmans BA. Progression of radiologic damage in patients with rheumatoid arthritis in clinical remission. Arthritis Rheum 2004;50:36-42.

6. Mäkinen H, Kautiainen H, Hannonen P, etal. Sustained remission and reduced radiographic progression with combination disease modifying antirheumatic drugs in early rheumatoid arthritis. J Rheumatol 2007;34:316-321. 
7. Peluso G, Michelutti A, Bosello S, Gremese E, Tolusso B, Ferraccioli G. Clinical and ultrasonographic remission determines different chances of relapse in early and long standing rheumatoid arthritis. Ann Rheum Dis 2011;70:172175.

8. Foltz V, Gandjbakhch F, Etchepare F, et al. Power Doppler Ultrasound, but Not Low-Field Magnetic Resonance Imaging, Predicts Relapse and Radiographic Disease Progression in Rheumatoid Arthritis Patients With Low Levels of Disease Activity. Arthritis Rheum 2012;64:67-76.

9. Scire CA, Montecucco C, Codullo V, Epis O, Todoerti M, Caporali R. Ultrasonographic evaluation of joint involvement in early rheumatoid arthritis in clinical remission: power Doppler signal predicts short-term relapse. Rheumatology 2009;48:1092-1097.

10. Saleem B, Brown AK, Keen H, et al. Disease remission state in patients treated with the combination of tumor necrosis factor blockade and methotrexate or with diseasemodifying antirheumatic drugs: A clinical and imaging comparative study. Arthritis Rheum 2009;60:1915-1922.

11. Saleem B, Brown AK, Quinn M, et al. Can flare be predicted in DMARD treated RA patients in remission, and is it important? A cohort study. Ann Rheum Dis 2012;71:13161321.

12. Dohn UM, Ejbjerg B, Boonen A, et al. No overall progression and occasional repair of erosions despite persistent inflammation in adalimumab-treated rheumatoid arthritis patients: results from a longitudinal comparative MRI, ultrasonography, CT and radiography study. Ann Rheum Dis 2011;70:252-258.

13. Wakefield RJ, Green MJ, Marzo-Ortega H, et al. Should oligoarthritis be reclassified? Ultrasound reveals a high prevalence of subclinical disease. Ann Rheum Dis 2004;63:382385.

14. Geng Y, Han J, Deng X, Zhang Z. Deep clinical remission: an optimized target in the management of rheumatoid arthritis? Experience from an ultrasonography study. Clin Exp Rheumatol 2016;34:581-586.

15. Wakefield RJ, Freeston JE, Hensor EM, Bryer D, Quinn MA, Emery P. Delay in imaging versus clinical response: a rationale for prolonged treatment with antitumor necrosis factor medication in early rheumatoid arthritis. Arthritis Rheum 2007;57:1564-1567.

16. Ozgocmen S, Ozdemir H, Kiris A, Bozgeyik Z, Ardicoglu O. Clinical evaluation and power Doppler sonography in rheumatoid arthritis: evidence for ongoing synovial inflammation in clinical remission. South Med J 2008;101:240245.

17. Saleem B, Brown AK, Keen H, et al. Should imaging be a component of rheumatoid arthritis remission criteria? A comparison between traditional and modified composite remission scores and imaging assessments. Ann Rheum Dis 2011;70:792-798.

18. Ten Cate DF, Luime JJ, Swen N, et al. Role of ultrasonography in diagnosing early rheumatoid arthritis and remission of rheumatoid arthritis - a systematic review of the literature. Arthritis Res Ther 2013;15:R4.
19. Janta I, Valor L, De la Torre I, et al. Ultrasound-detected activity in rheumatoid arthritis on methotrexate therapy: Which joints and tendons should be assessed to predict unstable remission? Rheumatol Int 2016;36:387-396.

20. Naredo E, Valor L, De la Torre I, et al. Ultrasound joint inflammation in rheumatoid arthritis in clinical remission: how many and which joints should be assessed? Arthritis Care Res (Hoboken) 2013;65:512-517.

21. Vlad V, Berghea F, Iorgoveanu V, Popescu M, Predeteanu $\mathrm{D}$, Ionescu R. Does patients' opinion of remission in rheumatoid arthritis overlap ultrasound "true" remission? - a pilot study. Med Ultrason 2018;20:328-334.

22. Möller I, Janta I,Backhaus M, et al. The 2017 EULAR standardised procedures for ultrasound imaging in rheumatology. Ann Rheum Dis 2017;76:1974-1979.

23. Colebatch AN, Edwards CJ, Ostergaard M, et al. EULAR recommendations for the use of imaging of the joints in the clinical management of rheumatoid arthritis. Ann Rheum Dis 2013;72:804-814.

24. Grassi W, Filippucci E, Farina A, Cervini C. Sonographic imaging of tendons. Arthritis Rheum 2000;43:969-976.

25. Ellegaard K, Torp-Pedersen S, Holm CC, DanneskioldSamsøe B, Bliddal H. Ultrasound in finger joints: findings in normal subjects and pitfalls in the diagnosis of synovial disease. Ultraschall Med 2007;28:401-408.

26. Millot F, Clavel G, Etchepare F, et al; Investigators of the French Early Arthritis Cohort ESPOIR. Musculoskeletal ultrasonography in healthy subjects and ultrasound criteria for early arthritis (the ESPOIR cohort). J Rheumatol 2011;38:613-620.

27. Szkudlarek M, Klarlund M, Narvestad E, et al. Ultrasonography of the metacarpophalangeal and proximal interphalangeal joints in rheumatoid arthritis: a comparison with magnetic resonance imaging, conventional radiography and clinical examination. Arthritis Res Ther 2006;8:R52.

28. Kitchen J, Kane D. Greyscale and power Doppler ultrasonographic evaluation of normal synovial joints: correlation with pro- and anti-inflammatory cytokines and angiogenic factors. Rheumatology (Oxford) 2015;54:458-462.

29. Parodi M, Silvestri E, Garlaschi G, Cimmino MA. How normal are the hands of normal controls? A study with dedicated magnetic resonance imaging. Clin Exp Rheumatol 2006;24:134-141.

30. Padovano I, Costantino F, Breban M, D'Agostino MA. Prevalence of ultrasound synovial inflammatory findings in healthy subjects. Ann Rheum Dis 2016;75:1819-1823.

31. Agten CA, Rosskopf AB, Jonczy M, Brunner F, Pfirrman CWA, Buck FM. Frequency of inflammatory-like MR imaging findings in asymptomatic fingers of healthy volunteers. Skeletal Radiol 2018;47:279-287.

32. Rowbotham EL, Freeston JE, Emery P, Grainger AJ. The prevalence of tenosynovitis of the interosseous tendons of the hand in patients with rheumatoid arthritis. Eur Radiol 2016;26:444-450.

33. Terslev L, Torp-Pedersen S, Qvistgaard E, von der Recke $\mathrm{P}$, Bliddal H. Doppler ultrasound findings in healthy wrists and finger joints. Ann Rheum Dis 2004;63:644-648. 
34. Ejbjerg B, Narvestad E, Rostrup E, et al. Magnetic resonance imaging of wrist and finger joints in healthy subjects occasionally shows changes resembling erosions and synovitis as seen in rheumatoid arthritis. Arthritis Rheum 2004;50:1097-1106.

35. Zufferey P, Möller B, Brulhart L, et al. Persistence of ultrasound synovitis in patients with rheumatoid arthritis fulfilling the DAS28 and/or the new ACR/ EULAR RA remission definitions: results of an observational cohort study. Joint Bone Spine 2014;81:426-432.

36. Micu MC, Fodor D, Micu R, Bolboacă SD, Ionescu R. Pregnant versus non-pregnant healthy subjects - a prospective longitudinal musculoskeletal ultrasound study concerning the spectrum of normality. Med Ultrason 2018;20:319327.

37. Tan AL, Grainger AJ, Tanner SF, et al. High-Resolution Magnetic Resonance Imaging for the Assessment of Hand Osteoarthritis. Arthritis Rheum 2005;52:2355-2365.

38. Wakefield RJ, Balint PV, Szudlarek M, et al. Musculoskeletal ultrasound including definitions for ultrasonographic pathology. J Rheumatol 2005;32:2485-2487.

39. Naredo E, d'Agostino MA, Wakefield RJ, et al; OMERACT Ultrasound Task Force. Reliability of a consensus based ultrasound score for tenosynovitis in rheumatoid arthritis. Ann Rheum Dis 2013;72:1328-1334.

40. Szkudlarek M, Court-Payen M, Jacobsen S, Klarlund M, Thomsen HS, Ostergaard M. Interobserver agreement in ultrasonography of the finger and toe joints in rheumatoid arthritis. Arthritis Rheum 2003;48:955-962.

41. Ostergaard M, Peterfy C, Conaghan P, et al. OMERACT rheumatoid arthritis magnetic resonance imaging studies. Core set of MRI acquisitions, joint pathology definitions, and the OMERACT RA-MRI scoring system. J Rheumatol 2003;30:1385-1386.

42. Haavardsholm EA, Ostergaard M, Ejbjerg BJ, Kvan NP, Kvien TK. Introduction of a novel magnetic resonance imaging tenosynovitis score for rheumatoid arthritis: reliability in a multireader longitudinal study. Ann Rheum Dis 2007;66:1216-1220.

43. Jäntschi L, Bolboacă SD. Exact Probabilities and Confidence Limits for Binomial Samples: Applied to the Difference between Two Proportions. ScientificWorldJournal 2010;10:865-878.
44. Navalho M, Resende C, Rodrigues AM, et al. Bilateral MR imaging of the hand and wrist in early and very early inflammatory arthritis: tenosynovitis is associated with progression to rheumatoid arthritis. Radiology 2012;264:823833.

45. Lindegaard HM, Vallø J, Hørslev-Petersen K, Junker P, Østergaard M. Low-cost, low-field dedicated extremity magnetic resonance imaging in early rheumatoid arthritis: a 1-year follow-up study. Ann Rheum Dis 2006;65:12081212.

46. McQueen F, Beckley V, Crabbe J, Robinson E, Yeoman S, Stewart N. Magnetic resonance imaging evidence of tendinopathy in early rheumatoid arthritis predicts tendon rupture at six years. Arthritis Rheum 2005;52:744-751.

47. Cader MZ, Filer A, Hazlehurst J, de Pablo P, Buckley CD, Raza K. Performance of the 2010 ACR/EULAR criteria for rheumatoid arthritis: comparison with 1987 ACR criteria in a very early synovitis cohort. Ann Rheum Dis 2011;70:949955.

48. Navalho M, Resende C, Rodrigues AM, et al. Bilateral evaluation of the hand and wrist in untreated early inflammatory arthritis: a comparative study of ultrasonography and magnetic resonance imaging. J Rheumatol 2013;40;1282-1292.

49. Wakefield RJ, O'Connor PJ, Conaghan PG, et al. Finger tendon disease in untreated early rheumatoid arthritis: a comparison of ultrasound and magnetic resonance imaging. Arthritis Rheum 2007;57:1158-1164.

50. Micu MC, Berghea F, Fodor D. Concepts in diagnosing, scoring, and monitoring tenosynovitis and other tendon abnormalities in patients with rheumatoid arthritis - the role of musculoskeletal ultrasound. Med Ultrason 2016;18:370377.

51. Fodor D, Felea I, Popescu D, et al. Ultrasonography of the metacarpophalangeal joints in healthy subjects using an 18 MHz transducer. Med Ultrason 2015;17:185-191.

52. Vreju FA, Filippucci E, Gutierrez M, et al. Subclinical ultrasound synovitis in a particular joint is associated with ultrasound evidence of bone erosions in the same joint in rheumatoid patients in clinical remission. Clin Exp Rheumatol 2016;34:673-678.

53. Radiology and Radiation Oncology/Imaging Technology News. Available at: www.itnonline.com. Last accessed 2018 October 5. 